\title{
Molecular Imaging in Tumor Angiogenesis and Relevant Drug Research
}

\author{
Xibo Ma, Jie Tian, Xin Yang, and Chenghu Qin \\ Intelligent Medical Research Center, Institute of Automation, Chinese Academy of Sciences, Beijing 100190, China \\ Correspondence should be addressed to Jie Tian, tian@ieee.org
}

Received 9 March 2011; Revised 16 May 2011; Accepted 23 May 2011

Academic Editor: Habib Zaidi

Copyright () 2011 Xibo Ma et al. This is an open access article distributed under the Creative Commons Attribution License, which permits unrestricted use, distribution, and reproduction in any medium, provided the original work is properly cited.

Molecular imaging, including fluorescence imaging (FMI), bioluminescence imaging (BLI), positron emission tomography (PET), single-photon emission-computed tomography (SPECT), and computed tomography (CT), has a pivotal role in the process of tumor and relevant drug research. CT, especially Micro-CT, can provide the anatomic information for a region of interest (ROI); PET and SPECT can provide functional information for the ROI. BLI and FMI can provide optical information for an ROI. Tumor angiogenesis and relevant drug development is a lengthy, high-risk, and costly process, in which a novel drug needs about 10-15 years of testing to obtain Federal Drug Association (FDA) approval. Molecular imaging can enhance the development process by understanding the tumor mechanisms and drug activity. In this paper, we focus on tumor angiogenesis, and we review the characteristics of molecular imaging modalities and their applications in tumor angiogenesis and relevant drug research.

\section{Introduction}

Drug development, especially antitumor drug development, is a relatively long process. Drug development contains five procedures, assessment of target expression, lead compound optimization, pre-Phase 1 studies, clinical Phase 1-3 studies, and Federal Drug Association (FDA) approval [13 ]. Angiogenesis is one of the fundamental characteristics during tumor progression and metastasis [4]. Detection of newborn tumor blood vessels and antiangiogenic drugs are being widely studied. Traditional biological techniques usually require euthanasia of experimental animals to acquire the correlative biological information of interest which make continuous detection impossible.

In recent years, molecular imaging emerged with increasing popularity in monitoring the changes at a molecular level in vivo and in evaluating treatment efficacy much earlier and much more accurately [5]. In contrast with traditional biological technology, molecular imaging can provide a whole body readout in an intact system through a longitudinal study in the same animals, provided that more statistically relevant and more accurate results are presented in evaluating the efficacy of antitumor drugs [6-8]. Because of the characteristics of molecular imaging, it can certainly decrease the workload and speed up drug development. In this paper, we review the characteristics and principles of different molecular imaging modalities before describing how molecular imaging can be used in tumor angiogenesis detection and relevant drug development.

\section{Characteristics and Principles of Molecular Imaging}

Molecular imaging can monitor biological processes at cellular and molecular levels in intact living subjects. Optical molecular imaging, such as bioluminescence imaging (BLI) and fluorescence imaging, has the advantage of high sensitivity and low cost. BLI uses light produced by the enzymatic reaction of a luciferase enzyme and its substrate. Based on this imaging principle, split-protein strategies were developed to assess protein-protein interactions in vivo using BLI techniques [9-19]. By detecting the bioluminescence photons, we can characterize a variety of cellular and molecular processes, including protein interactions [20-23], protein degradation [24-26], and protease activity [27-29]. Otherwise, the firefly luciferase gene could be transferred into tumor cells and could be used to evaluate the tumor response 


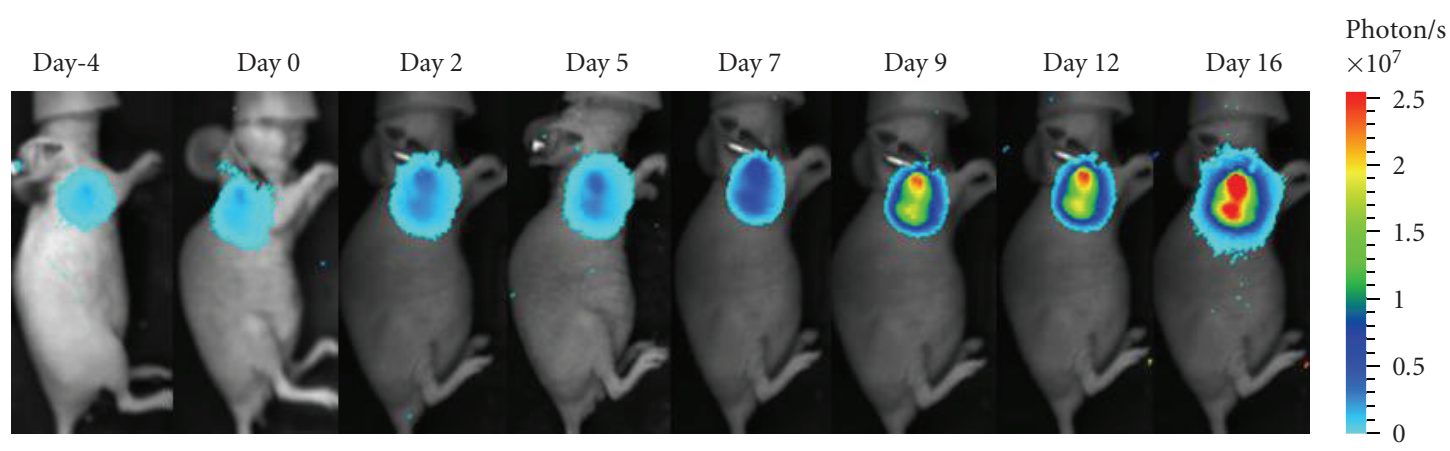

(a)

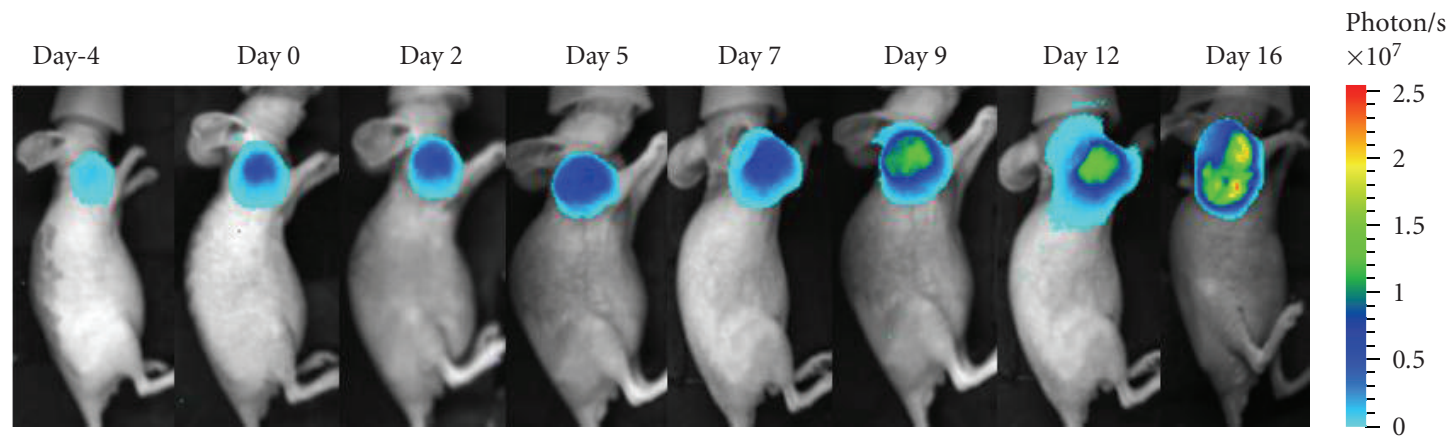

(b)

FIGURE 1: Serial bioluminescence images of the HCC-LM3-fLuc tumor-bearing nude mice that underwent saline (a) or cyclophosphamide (b) treatment (data from Ma et al. [6]).

to chemotherapeutic agents in living animals. In Figure 1 [6], we used BLI to evaluate the antitumor efficacy of cyclophosphamide in HCC-LM3-fLuc tumor-bearing nude mice.

After imaging acquisition, we could count the photons in a region of interest (ROI) to quantify the antitumor efficacy by using WINMI (windows-based molecular imaging), which was developed based on the medical imaging ToolKit (MITK [30]; Medical Image Processing and Analyzing group, Institute of Automation, Chinese Academy of Sciences, Beijing, China; http://www.mitk.net/).

The tumor volume was measured using a calendar in order to evaluate the antitumor efficacy of the drug. However, conventional measurement with a calendar usually introduced more induced errors. Through quantification of the light intensity, this BLI technique can provide more accurate information of tumor progression. However, this technique can only provide two-dimensional information on tumor which cannot fulfill the need for inner tumor detection and corresponding drug evaluation. Furthermore, when combined with computed tomography (CT), we have developed our prototype system to detect inner tumors in the mouse. The dual modality system (Micro-CT and BLI) is shown in Figure 2. Micro-CT can provide threedimensional anatomic structure information to form the heterogeneous mouse which is proven to be more accurate in reconstructing the three-dimensional location information [31]. This technique provides an effective tool for inner tumor detection and evaluation of antitumor drugs.
In fluorescence imaging (FMI), an external light with an appropriate wavelength was used to excite a target fluorescent molecule. Then, almost immediately, the target fluorescent molecules released a longer wavelength, lower-energy light for imaging. In contrast to BLI, the detection depth of FMI is greater than a few millimeters by using the near infrared fluorescence (NIRF) probe and dye [8]. After imaging acquisition and filtering of the background noises, the highcontrast images can be obtained. Using the same method with BLI, the light intensity can be counted to evaluate the tumor volume and antitumor drug efficacy.

Positron emission tomography (PET) and single-photon emission-computed tomography (SPECT) are imaging modalities that are widely used in preclinical and clinical studies $[32,33]$. They can be used to detect the molecular and cellular changes of diseases before the tumor is large enough to cause structural changes by injecting radiotracer labeled probes $[34,35]$ ( such as ${ }^{18} \mathrm{~F}$ for PET, ${ }^{131}$ I for SPECT). These imaging modalities have been applied not only in rodents or larger animals, but also in clinical trials, such as for disease diagnosis [34] and monitored disease therapy [36]. Combined with morphological/anatomical imaging modalities (such as CT), PET/SPECT can be used to obtain threedimensional tomography images and can provide dynamic functional information. Because of the decay characteristics of a radionuclide, time-decay calibration was often used to attain radionuclide uptake per weight for the comparison between different tissues. In contrast with BLI/FMI, PET/SPECT shares the advantage of having high sensitivity 


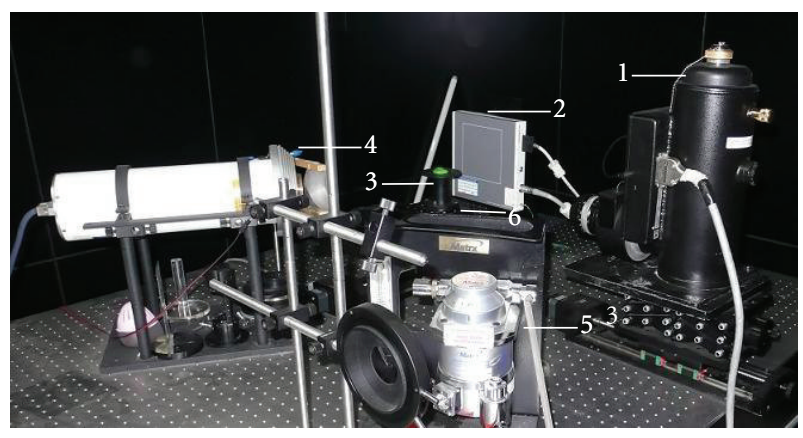

FIGURE 2: Our prototype BLT/Micro-CT dual modality imaging system. (1) CCD camera; (2) X-ray detector; (3) mouse holder; (4) Xray tube; (5) anesthesia machine; (6) rotation stage.

with unlimited depth penetration. However, they also share the limitations of high cost and limited spatial resolution.

CT is widely used in diagnostic medicine as it enables doctors to provide safe, cross-sectional images with a high resolution to patients. It uses $\mathrm{X}$-rays for forming tissue images, and the images are reconstructed from its projections. A projection means a line integral to the X-ray attenuation coefficients of the images in a given direction. In preclinical research, Micro-CT for small animal imaging has played a critical role in the evolution of molecular imaging [37]. The X-Ray data was reconstructed based on cone-beam geometry utilizing a Feldkamp-Davis-Kress (FDK) algorithm [38], which is an approximate algorithm, and the cone angle should be limited to a relatively small value to avoid cone-beam artifacts. Micro-CT can obtain high resolution anatomic information which can integrate with other modalities, such as nuclear and optical approaches. Since X-ray attenuation of soft tissues is different from that of bones, Micro-CT is very suitable for bone detection, such as bone growth, destruction, and changes in bone density. Despite lacking a targeted contrast agent, Micro-CT is not able to acquire molecular imaging directly, but it can indirectly reflect the changes of cells and molecular structures, such as imaging changes in vascular density [39]. For in vivo imaging, especially in a longitudinal study, the radiation dose should be considered, and much attention has been paid to CT imaging with a high resolution as well as a low-radiation dose.

\section{Tumor Angiogenesis}

Tumor angiogenesis is a process of proliferation of a network of blood vessels that penetrates into cancerous growths, supplying nutrients and oxygen and removing waste products. Tumor angiogenesis is activated by the signal molecules that are sent by cancerous tumor cells. These signals activate certain genes in the host tissue, make proteins, and form the network of new blood vessels surrounding the tumor. These signal molecules include, but are not limited to, growth factor receptors, tyrosine kinase receptors, integrins, and matrix metalloproteinases [40] (MMP). In this paper, we will focus on two of the most intensively studied angiogenesisrelated molecular targets: vascular endothelial growth factor (VEGF) and VEGF receptors (VEGFR), and integrin $\alpha_{v} \beta_{3}$. During tumor angiogenesis, these molecules can be used as indicators in molecular imaging. By targeting them, we can diagnose some tumors which express these molecules. Furthermore, we can evaluate the antitumor efficacy of drugs using the correlative probes.

\section{Imaging VEGF/VEGFR Expression}

VEGF is a powerful mitogen in embryonic and somatic angiogenesis. It plays a pivotal role in both normal vascular tissue development and tumor neovascularization. Overexpression of VEGF is associated with tumor progression and poor prognosis in several tumors, including colorectal, gastric, and pancreatic carcinomas; angiosarcomas; breast, prostate, and lung cancers; malignant gliomas; melanoma.

Through building a transgenic mouse of a VEGF promoter driven GFP reporter gene, Izzo's group proved fluorescence imaging is credible in detecting the VEGF [41]. Afterwards, Wang's group used BLI to detect VEGF in a transgenic mouse model [42]. Vascular endothelial growth factor A (VEGFR-1) and Flk-1/KDR (VEGFR-2) are key regulators for tumor angiogenesis and tumor growth. Chen's group monitored the antiangiogenic and antitumor efficacies of a vasculature-targeting fusion toxin $\left(\mathrm{VEGF}_{121} / \mathrm{rGel}\right) \mathrm{com}$ posed of the VEGF-A isoform VEGF 121 linked with a $\mathrm{G}_{4} \mathrm{~S}$ tether to recombinant plant toxin gelonin $(\mathrm{rGel})$ in an orthotopic glioblastoma mouse model by use of noninvasive in vivo BLI, magnetic resonance imaging (MRI), and PET. Through this study, they proved multimodality imaging and therapy with $\mathrm{VEGF}_{121} / \mathrm{rGel}$ could provide an effective means to prospectively identify patients who will benefit from $\mathrm{VEGF}_{121} / \mathrm{rGel}$ therapy and then stratify, personalize, and monitor treatment to obtain optimal survival outcomes in future clinical applications [43].

As an effective imaging technique, PET has been applied in the clinical studies. With the development of Micro-PET scanners dedicated to small animal imaging studies, it can provide a similar in vivo imaging capability in mice, rats, monkeys, and humans $[44,45]$. As an IgG1 monoclonal antibody that binds to human VEGF, VG76e was labeled with ${ }^{124}$ I for PET imaging of solid tumor xenografts in immunedeficient mice [46]. Chen's group [47] labeled $\mathrm{VEGF}_{121}$ with ${ }^{64} \mathrm{Cu}$ for PET imaging of tumor angiogenesis and VEGFR expression. This study showed that ${ }^{64} \mathrm{Cu}$-DOTA$\operatorname{VEGF}_{121}(\sim 15 \% \mathrm{ID} / \mathrm{g})$ had a rapid, specific, and prominent uptake in highly vascularized small U87MG tumors with high VEGFR-2 expression but lower and sporadic uptake in large U87MG tumors with low VEGFR-2 expression [47]. In a follow-up study, a VEGFR-2 specific fusion toxin $\mathrm{VEGF}_{121} / \mathrm{rGel}$ (composed of $\mathrm{VEGF}_{121}$ linked with a $\mathrm{G}_{4} \mathrm{~S}$ tether to the recombinant plant toxin gelonin) was used to treat orthotopic glioblastoma in a mouse model [48]. In addition, recombinant human VEGF $_{121}$ was labeled with ${ }^{111}$ In for SPECT imaging to identify ischemic tissue in a rabbit model, where unilateral hind-limb ischemia was created 
by femoral artery excision [49]. Furthermore, VEGF ${ }_{121}$ has also been labeled with ${ }^{99 \mathrm{~m}} \mathrm{Tc}$ through an "adapter/docking" strategy, and the tracer was tested in a 4T1 murine mammary carcinoma with tumor uptake of about $3 \% \mathrm{ID} / \mathrm{g}[50,51]$.

\section{Imaging $\alpha_{v} \beta_{3}$ Expression}

$\alpha_{v} \beta_{3}$ is a member of the integrin family which is a kind of adhesion molecule consisting of two noncovalently bound transmembrane subunits ( $\alpha$ and $\beta$ ). In mammals, $18 \alpha(120$ $185 \mathrm{KD})$ and $9 \beta(90-110 \mathrm{kd})$ subunits assemble into 24 kinds of different receptors. Integrin signaling pathways play a crucial role in tumor angiogenesis and metastasis. Tumor growth, angiogenesis, and metastasis can be inhibited by blocking integrin signaling. Integrin $\alpha_{v} \beta_{3}$ is an important indicator in evaluating the antitumor efficacy of drugs to the $\alpha_{v} \beta_{3}$ positive tumors. Some antibodies, peptides, peptidomimetics, and other antagonists have been proven to have great potential in the treatment of cancer.

Integrin $\alpha_{v} \beta_{3}$ is often expressed on some tumor cells in the brain, such as glioblastoma multiforme, and striatum neutral stem-cells-derived primary tumor. Via radiotracer labeling of some ligands or antibodies, the expression of integrin $\alpha_{v} \beta_{3}$ can be imaged by Micro-PET or SPECT [52]. In Jia's research [53], a ${ }^{99 \mathrm{~m}}$ Tc-Labeled cyclic RGDfK dimer has been studied to evaluate its application for SPECT imaging of glioma integrin $\alpha_{v} \beta_{3}$ expression. They found that glioma tumors could be clearly visualized with all three radiotracers at $4 \mathrm{~h}$ after injection and $99 \mathrm{mTc}(\mathrm{SQ} 168)$ (tricine)(TPPTS) (*) [SQ168) [2-[[[5-[carboonyl]-2-pyridinyl]-hydrazono] methyl] benz-enesulfonic acid]-Glu(cyclo\{Lys-Arg-GlyAsp-D-Phe \})-cyclo\{Lys-Arg-Gly-Asp-D-Phe\}) had the best imaging quality among the three radiotracers [53] $\left(\left[{ }^{99 \mathrm{~m}} \mathrm{Tc}(\mathrm{SQ} 168)(\mathrm{EDDA})\right], \quad\left[{ }^{99 \mathrm{~m}} \mathrm{Tc}(\mathrm{SQ} 168)\right.\right.$ (tricine)(PDA)], and $\left[{ }^{99 \mathrm{~m}} \mathrm{Tc}(\mathrm{SQ} 168)(\right.$ tricine $\left.)(\mathrm{TPPTS})\right] \quad(\mathrm{SQ} 168=[2-[[[5-$ [carboonyl]-2-pyridinyl]-hydrazono]methyl]benzenesulfonic acid]-Glu (cyclo\{Lys-Arg-Gly-Asp-D-Phe $\}$ )-cyclo\{LysArg-Gly-Asp-D-Phe $\}$; EDDA = ethylenediamine- $N, N^{\prime}$ diacetic acid; PDA = 2,5-pyridinedicarboxylic acid; TPPTS = trisodium triphenylphosphine-3,3',3"'-trisulfonate). ${ }^{99 m} \mathrm{Tc}$ labeled RGD has also been used in gamma imaging of secondary tumors of transplanted human fetal striatal neutral stem-cells-derived primary tumor cells [54]. The results showed both PET and SPECT could be used in imaging tumorigenesis and metastasis of integrin $\alpha_{v} \beta_{3}$ positive tumors. Some other radiotracers included ${ }^{18} \mathrm{~F}$ $[55,56],{ }^{68} \mathrm{Ga}-[57]$, and ${ }^{64} \mathrm{Cu}-[58]$ labeled RGD that were applied in imaging the integrin $\alpha_{v} \beta_{3}$ expression using PET or SPECT. All of the radiotracers also validated the potential value in preclinical and clinical studies. In Cao's research [58], ${ }^{64} \mathrm{Cu}$-labeled RGD was used in imaging teratoma formation derived from hES cells by targeting $\alpha_{v} \beta_{3}$ integrin in a nude mouse. Furthermore, by comparing it to BLI, ${ }^{64} \mathrm{Cu}$ DOTA-RGD4 proved to have the ability of noninvasively visualizing teratoma formation in vivo. Compared with the two other probes, ${ }^{18} \mathrm{~F}$-labeled FLT and 18F-Labeled FDG, ${ }^{64} \mathrm{Cu}-\mathrm{DOTA}-\mathrm{RGD} 4$ has a higher sensitivity and tumor to background contrast (data shown in Figure 3). ${ }^{90} \mathrm{Y}-[59]$ and
${ }^{111}$ IN-[60] labeled monoclonal antibody abegrin had also been studied in imaging $\alpha_{v} \beta_{3}$ expression of U87 glioblastoma multiforme.

Otherwise, fluorescent dye-labeled E $\left[\mathrm{PEG}_{4}-\mathrm{c}(\mathrm{RGDfK})\right]_{2}$ had been developed to image integrin $\alpha_{v} \beta_{3}$ expression by Liu and his coworkers [61]. By comparing the characterization of dye-labeled E $\left[\mathrm{PEG}_{4}-\mathrm{c}(\mathrm{RGDfK})\right]_{2}$ with dye-labeled RGD dimer without $\mathrm{PEG}_{4}$ linkers, Cy5.5-labeled E $\left[\mathrm{PEG}_{4}{ }^{-}\right.$ $\mathrm{c}(\mathrm{RGDfK})]_{2}$ showed higher tumor accumulation and tumorto-background contrast (data shown in Figure 4). In vivo NIRF imaging with IRDye800 E $\left[\mathrm{PEG}_{4}-\mathrm{c}(\mathrm{RGDfK})\right]_{2}$ was confirmed to offer an easy, fast, and low-cost way to detect and semiquantify tumor integrin $\alpha_{v} \beta_{3}$ expression in living subjects.

Integrin $\alpha_{v} \beta_{3}$ is one of the most extensively studied molecular targets involved in tumor angiogenesis [62-65]. Markiewicz's group had discovered a presumably inactive linear hexapeptide GRDSPK with near-IR carbocyanine molecular probe yielding Cyp-GRD which could be used to target integrin $\alpha_{v} \beta_{3}$-positive tumors [66]. They later synthesized and evaluated a series of multimeric RGD compounds constructed on a dicarboxylic acid-containing near-IR fluorescent dye cypate for tumor targeting [67]. Furthermore, optimization of the spatial alignment and ligand reconstructed of the RGD moieties through careful molecular design may induce multivalent ligand-receptor interactions useful for in vivo tumor imaging and tumor-targeted therapy. As a humanized monoclonal antibody against human integrin $\alpha_{v} \beta_{3}$ (picomolar binding affinity), abegrin (MEDI522, also called Vitaxin; MedImmune, Inc., Gaithersburg, $\mathrm{Md}$ ) is used in clinical trials for cancer therapy. Tice's group had conjugated abegrin with macrocyclic chelating agent DOTA and labeled it with ${ }^{64} \mathrm{Cu}$ for PET imaging of a tumor xenograft [68]. Numerous reports on multimodality molecular imaging of integrin $\alpha_{v} \beta_{3}$ showed that tumor angiogenesis imaging can participate in multiple stages of the drug development process, including target validation, lead compound optimization, compound screening, and clinical trials.

\section{Conclusion and Perspective}

Molecular imaging has been widely applied in preclinical studies using some radiotracer probes, and PET and SPECT have been used in clinical diagnoses and antitumor evaluation of some drugs. However, optical molecular imaging especially BLI can only be used in preclinical studies. Many problems can be solved by applying molecular imaging in clinical research.

BLI has more widely used applications in tumor detection and evaluation of pharmacodynamics, toxicity, and pharmacokinetics because of its noninvasive molecular and cellular level detection ability, high sensitivity, and low cost in comparison with other imaging technologies. However, BLI cannot present the accurate location and intensity of the inner bioluminescent sources such as in the bone, liver, or lungs. Bioluminescent tomography (BLT) shows its advantages in determining the bioluminescent source distribution inside a small animal or phantom. By utilizing CT 


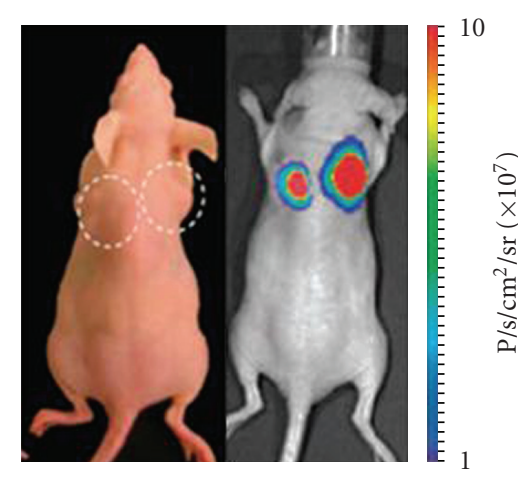

(a)

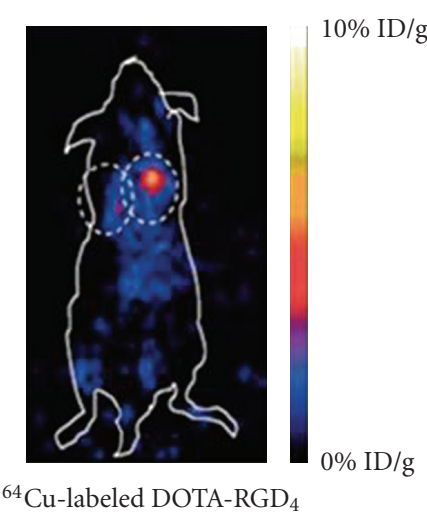

(b)

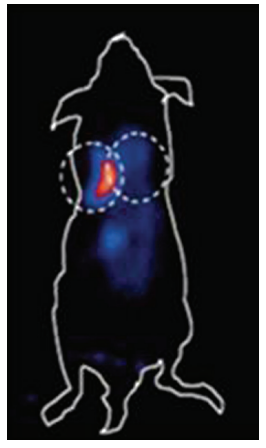

${ }^{18}$ F-labeled FLT

(c)

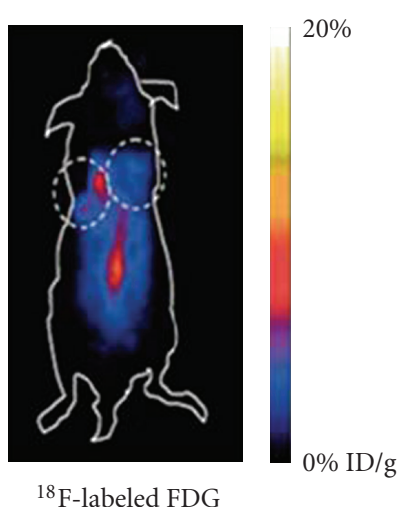

(d)

Figure 3: Micro-PET imaging of ${ }^{18} \mathrm{~F}-\mathrm{FDG},{ }^{18} \mathrm{~F}-\mathrm{FLT}$, and ${ }^{64} \mathrm{Cu}-\mathrm{DOTA}-\mathrm{RGD} 4$ on hES cell-derived teratoma. (a) Background imaging and bioluminescence imaging show teratoma formation in H9Hes-DF cells in the right shoulder and tumor formation in the control 2008 cell line in the left shoulder $\left(7.3 \pm 2.5 \times 10^{7}\right.$ versus $4.5 \pm 1.6 \times 10^{7}$ photon $/ \mathrm{s} / \mathrm{cm}^{2} / \mathrm{sr}$, resp.); (b) specific and prominent uptake of ${ }^{64} \mathrm{Cu}-\mathrm{DOTA}$ RGD4 was found in the vascularized teratoma but not in the control human ovarian carcinoma 2008 cell line with low integrin expression $(P<0.01)$; (c) hES cell-derived teratomas had a low uptake of ${ }^{18} \mathrm{~F}$-FLT, whereas the 2008 cancer xenografts exhibited high ${ }^{18} \mathrm{~F}-\mathrm{FDG}$ and ${ }^{18}$ F-FLT uptakes; (d) hES cell-derived teratomas had a low uptake of ${ }^{18} \mathrm{~F}-\mathrm{FDG}$, whereas the 2008 cancer xenografts and abdomen exhibited high ${ }^{18}$ F-FDG and ${ }^{18}$ F-FLT uptakes (data from Cao et al. [58]).

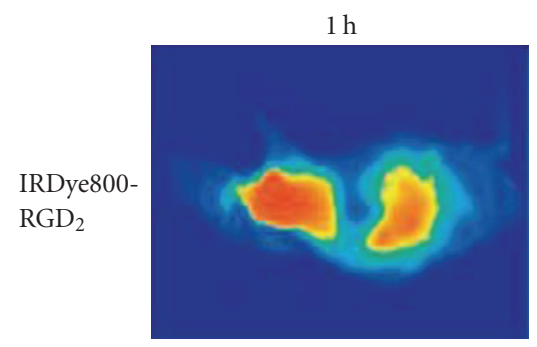

$1 \mathrm{~h}$

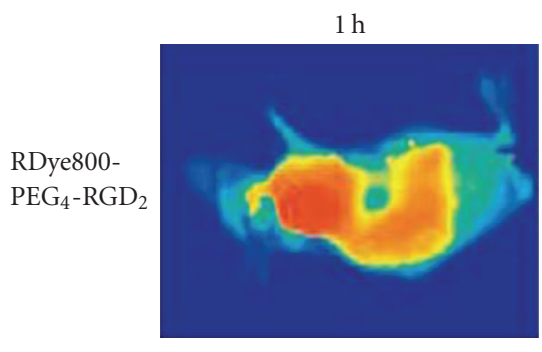

$2 \mathrm{~h}$

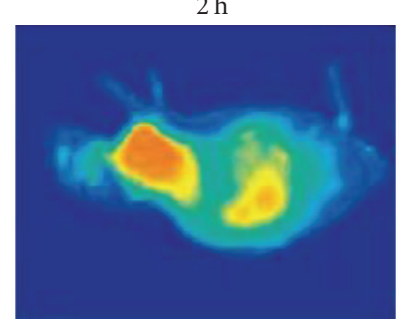

(a)

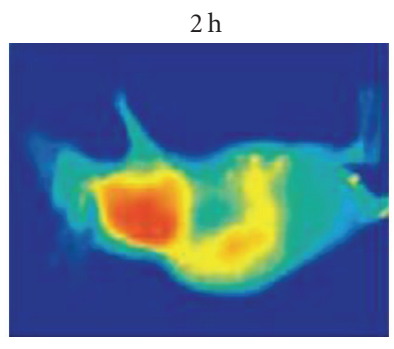

(b)
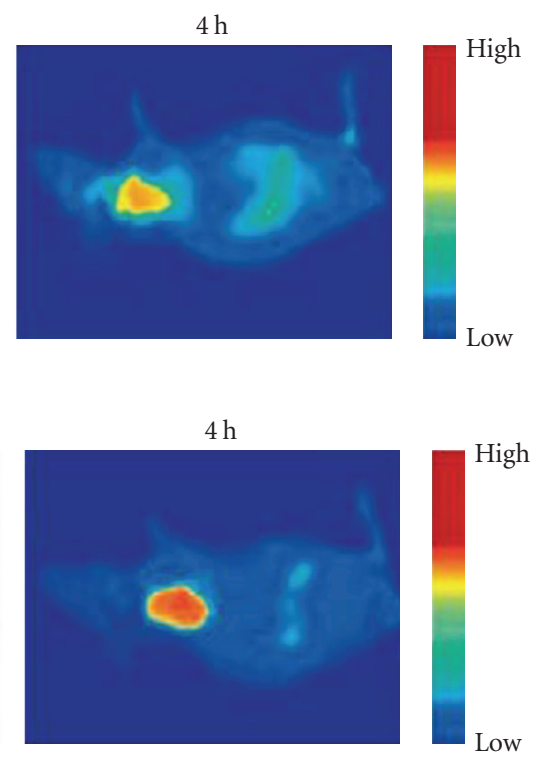

FIGURE 4: In vivo fluorescence imaging of U87MG tumor-bearing nude mice at 1, 2, and 4 hours after intravenous injection of 0.5 nmol IRDye800-RGD2 or IRDye800-PEG4-RGD2. Fluorescence signal from IRDye800 is pseudocolored (data from Liu et al. [61]).

information acquired by an X-ray detector, the three-dimensional location can be reconstructed using some BLT reconstruction methods such as the adaptive finite element method and Bayesian method These tomography imaging methods can be used in early detection of tumors [69] and assist in the diagnosis and evaluation of drug efficacy more accurately. Furthermore, the clinical application of BLI needs to develop some novel probes which can be used in humans. Developing a novel imaging probe is an expensive, timeconsuming procedure.
For PET/SPECT imaging, some radiotracers such as ${ }^{18} \mathrm{~F}$, ${ }^{64} \mathrm{Cu},{ }^{111} \mathrm{In}$, and ${ }^{68} \mathrm{Ga}$ have been used in labeling ligands and antibodies to image their relative receptors. However, the binding ability between some radiotracers, their ligands, and antibodies is low. Some single-targeted probes have poor pharmacokinetic properties with fast clearance speed. Furthermore, with the development of molecular imaging, some new dual-modality or multimodality probes need to be developed to acquire more general, more accurate information of the disease. In recent years, significant advances have 
been made in developing novel probes for multimodality molecular imaging of tumor angiogenesis. Among all of the studies regarding multimodality probes, multireceptortargeted imaging probes are most popular with some advantages. First of all, because some receptors may not be sufficiently expressed in all tumor cells during tumor progression and processes, with multireceptor targeting, more probes can accumulate in tumor cells and tissues. Secondly, the binding affinity of multireceptor-targeted probes can be relatively higher than in single receptor targeted probes. Thirdly, the clearance properties and excretion rates of dualor multireceptor-targeted probes may be optimal compared to single receptor-targeted probes. In general, developing novel, high-affinity probes is necessary for molecular imaging.

In summary, although imaging to tumor angiogenetic and relevant drugs have been reported in previous work, tumor angiogenesis and relevant drug studies are subject to many problems, such that a good probe for imaging VEGF/ VEGFR is limited which greatly hinders the development of imaging; imaging sensitivity needs to be improved which can help in determining whether or not to start and when to start VEGFR-targeted treatment. Accordingly, research on the following two aspects need to be strengthened in the future.

Based on the current probe, the development of a moresensitive, less-toxic multimodal molecular imaging probe is necessary.

Based on the current imaging technique, the development of more accurate image processing algorithms is the key to VEGF/VEGFR and relevant drug studies.

\section{Acknowledgments}

This paper is supported by the National Basic Research Program of China (973 Program) under Grant no. 2011CB707700, the Knowledge Innovation Project of the Chinese Academy of Sciences under Grant no. KGCX2YW-907, the National Natural Science Foundation of China under Grant nos. 81027002, 81071205, 30970778, the Science and Technology Key Project of Beijing Municipal Education Commission under Grant no. KZ200910005005, and the Fellowship for Young International Scientists of the Chinese Academy of Sciences under Grant no. 2010Y2GA03.

\section{References}

[1] J. K. Willmann, N. Bruggen, L. M. Dinkelborg, and S. S. Gambhir, "Molecular imaging in drug development," Nature Reviews Drug Discovery, vol. 7, no. 7, pp. 591-606, 2008.

[2] R. J. Hargreaves, "The role of molecular imaging in drug discovery and development," Clinical Pharmaclology \& Therapeutics, vol. 82, no. 2, pp. 349-353, 2007.

[3] A. Iagaru, X. Chen, and S. S. Gambhir, "Molecular imaging can accelerate anti-angiogenic drug development and testing," Nature Clinical Practice Oncology, vol. 4, no. 10, pp. 556-557, 2007.

[4] D. Hanahan and R. A. Weinberg, "The hallmarks of cancer," Cell, vol. 100, no. 1, pp. 57-70, 2000.
[5] W. Cai, J. Rao, S. S. Gambhir, and X. Chen, "How molecular imaging is speeding up antiangiogenic drug development," Molecular Cancer Therapeutics, vol. 5, no. 11, pp. 2624-2633, 2006.

[6] X. B. Ma, Z. F. Liu, and X. Yang, "Dual-modality monitoring of tumor response to cyclophosphamide therapy in mice with bioluminescence imaging and small-animal positron emission tomography," Molecular Imaging, vol. 10, no. 4, pp. 278-283, 2011.

[7] H. Prenen, C. Stefan, and A. Oosterom, "Imatinib mesylate inhibits glucose uptake in gastrointestinal stromal tumor cells by downregulation of the glucose transporters recruitment to the plasma membrane," American Journal of Biochemistry and Biotechnology, vol. 1, no. 2, pp. 95-102, 2005.

[8] G. D. Luker and K. E. Luker, "Optical imaging: current applications and future directions," Journal of Nuclear Medicine, vol. 49, no. 1, pp. 1-4, 2008.

[9] K. E. Luker and D. Piwnica-Worms, "Optimizing luciferase protein fragment complementation for bioluminescent imaging of protein-protein interactions in live cells and animals," Methods in Enzymology, vol. 385, pp. 349-360, 2004.

[10] T. F. Massoud, R. Paulmurugan, P. Ray, and S. S. Gambhir, "Reporter gene imaging of protein-protein interactions in living subjects," Current Opinion in Biotechnology, vol. 18, no. 1, pp. 31-37, 2007.

[11] T. F. Massoub, R. Paulmurugan, and S. S. Gambhir, "Molecular imaging of homodimeric protein-protein interactions in living subjects," Federation of American Societies for Experimental Biology Journal, vol. 18, no. 10, pp. 1105-1107, 2004.

[12] R. Paulmurugan and S. S. Gambhir, "An intramolecular folding sensor for imaging estrogen receptor-ligand interactions," Proceedings of the National Academy of Sciences of the United States of America, vol. 103, no. 43, pp. 15883-15888, 2006.

[13] R. Paulmurugan and S. S. Gambhir, "Combinatorial library screening for developing an improved split-firefly luciferase fragment-assisted complementation system for studying protein-protein interactions," Analytical Chemistry, vol. 79, no. 6, pp. 2346-2353, 2007.

[14] R. Paulmurugan, T. F. Massoud, J. Huang, and S. S. Gambhir, "Molecular imaging of drug-modulated protein-protein interactions in living subjects," Cancer Research, vol. 64, no. 6, pp. 2113-2119, 2004.

[15] R. Paulmurugan, P. Ray, A. De, C. T. Chan, and S. S. Gambhir, "Imaging protein-protein interactions in living animals," in Protein-Protein Interactions: A Molecular Cloning Manual, E. Golemis and P. D. Adams, Eds., vol. 1, pp. 695-713, Cold Spring Harbor Laboratory Press, New York, NY, USA, 2nd edition, 2005.

[16] R. Paulmurugan, P. Ray, A. De, C. T. Chan, and S. S. Gambhir, "Imaging protein-protein interactions in living subjects," Trends in Analytical Chemistry, vol. 24, no. 5, pp. 446-458, 2005.

[17] R. Paulmurugan, Y. Umezawa, and S. S. Gambhir, "Noninvasive imaging of protein-protein interactions in living subjects by using reporter protein complementation and reconstitution strategies," Proceedings of the National Academy of Sciences of the United States of America, vol. 99, no. 24, pp. 1560815613, 2002.

[18] G. D. Luker, V. Sharma, and D. W. Piwnica, "Visualizing protein-protein interactions in living animals," Methods, vol. 29, no. 1, pp. 110-122, 2003.

[19] V. Villalobos, S. Naik, and D. Piwnica-Worms, "Current state of imaging protein-protein interactions in vivo with 
genetically encoded reporters," Annual Review of Biomedical Engineering, vol. 9, pp. 321-349, 2007.

[20] R. Paulmurugant and S. S. Gambhir, "Monitoring proteinprotein interactions using split synthetic renilla luciferase protein-fragment-assisted complementation," Analytical Chemistry, vol. 75, no. 7, pp. 1584-1589, 2003.

[21] K. E. Luker, M. C. P. Smith, G. D. Luker, S. T. Gammon, H. Piwnica-Worms, and D. Piwnica-Worms, "Kinetics of regulated protein-protein interactions revealed with firefly luciferase complementation imaging in cells and living animals," Proceedings of the National Academy of Sciences of the United States of America, vol. 101, no. 33, pp. 12288-12293, 2004.

[22] R. Paulmurugan and S. S. Gambhir, "Novel fusion protein approach for efficient high-throughput screening of small molecule-mediating protein-protein interactions in cells and living animals," Cancer Research, vol. 65, no. 16, pp. 74137420, 2005.

[23] I. Remy and S. W. Michnick, "A highly sensitive proteinprotein interaction assay based on Gaussia luciferase," Nature Methods, vol. 3, no. 12, pp. 977-979, 2006.

[24] G. D. Luker, C. M. Pica, J. Song, K. E. Luker, and D. PiwnicaWorms, "Imaging 26S proteasome activity and inhibition in living mice," Nature Medicine, vol. 9, no. 7, pp. 969-973, 2003.

[25] G. J. Zhang, M. Safran, W. Wei et al., "Bioluminescent imaging of Cdk2 inhibition in vivo," Nature Medicine, vol. 10, no. 6, pp. 643-648, 2004.

[26] M. Safran, W. Y. Kim, F. O’Connell et al., "Mouse model for noninvasive imaging of HIF prolyl hydroxylase activity: assessment of an oral agent that stimulates erythropoietin production," Proceedings of the National Academy of Sciences of the United States of America, vol. 103, no. 1, pp. 105-110, 2006.

[27] M. Funovics, R. Weissleder, and C. H. Tung, "Protease sensors for bioimaging," Analytical and Bioanalytical Chemistry, vol. 377, no. 6, pp. 956-963, 2003.

[28] J. Grimm, D. G. Kirsch, S. D. Windsor et al., "Use of gene expression profiling to direct in vivo molecular imaging of lung cancer," Proceedings of the National Academy of Sciences of the United States of America, vol. 102, no. 40, pp. 14404-14409, 2005.

[29] T. Jiang, E. S. Olson, Q. T. Nguyen, M. Roy, P. A. Jennings, and R. Y. Tsien, "Tumor imaging by means of proteolytic activation of cell-penetrating peptides," Proceedings of the National Academy of Sciences of the United States of America, vol. 101, no. 51, pp. 17867-17872, 2004.

[30] J. Tian, J. Xue, Y. Dai, J. Chen, and J. Zheng, "A novel software platform for medical image processing and analyzing," IEEE Transactions on Information Technology in Biomedicine, vol. 12, no. 6, pp. 800-812, 2008.

[31] Y. Lv, J. Tian, W. Cong et al., "A multilevel adaptive finite element algorithm for bioluminescence tomography," Optics Express, vol. 14, no. 18, pp. 8211-8223, 2006.

[32] S. S. Gambhir, "Molecular imaging of cancer with positron emission tomography," Nature Reviews Cancer, vol. 2, no. 9, pp. 683-693, 2002.

[33] E. Bombardieri, "The added value of metabolic imaging with FDG-PET in oesophageal cancer: prognostic role and prediction of response to treatment," European Journal of Nuclear Medicine and Molecular Imaging, vol. 33, no. 7, pp. 753-758, 2006.

[34] T. Belhocine, N. Steinmetz, R. Hustinx et al., "Increased uptake of the apoptosis-imaging agent ${ }^{(99 \mathrm{~m})} \mathrm{Tc}$ recombinant human annexin $\mathrm{V}$ in human tumors after one course of chemotherapy as a predictor of tumor response and patient prognosis," Clinical Cancer Research, vol. 8, no. 9, pp. 27662774, 2002.

[35] H. H. Boersma, B. L. J. H. Kietselaer, L. M. L. Stolk et al., "Past, present, and future of annexin A5: from protein discovery to clinical applications," Journal of Nuclear Medicine, vol. 46, no. 12, pp. 2035-2050, 2005.

[36] L. Wang, J. Shi, Y. S. Kim et al., "Improving tumor-targeting capability and pharmacokinetics of ${ }^{99}$ Tc-labeled cyclic RGD dimers with PEG4 linkers," Molecular Pharmaceutics, vol. 6, no. 1, pp. 231-245, 2009.

[37] S. R. Cherry, "In vivo molecular and genomic imaging: new challenges for imaging physics," Physics in Medicine and Biology, vol. 49, no. 3, pp. R13-R48, 2004.

[38] L. A. Feldkamp, L. C. Davis, and J. W. Kress, "Practical conebeam algorithm," Journal of the Optical Society of America A, vol. 1, no. 6, pp. 612-619, 1984.

[39] E. L. Ritman, "Molecular imaging in small animals roles for micro-CT," Journal of Cellular Biochemistry, no. 39, pp. 116124, 2002.

[40] N. Ferrara, "VEGF and the quest for tumour angiogenesis factors," Nature Reviews Cancer, vol. 2, no. 10, pp. 795-803, 2002.

[41] A. D. Izzo, M. A. Mackanos, J. T. Beckham, and E. D. Jansen, "In vivo optical imaging of expression of vascular endothelial growth factor following laser incision in skin," Lasers in Surgery and Medicine, vol. 29, no. 4, pp. 343-350, 2001.

[42] Y. Wang, M. Iyer, A. Annala, L. Wu, M. Carey, and S. S. Gambhir, "Noninvasive indirect imaging of vascular endothelial growth factor gene expression using bioluminescence imaging in living transgenic mice," Physiological Genomics, vol. 24, no. 2, pp. 173-180, 2006.

[43] A. R. Hsu, W. B. Cai, A. Veeravagu et al., "Multimodality molecular imaging of glioblastoma growth inhibition with vasculature-targeting fusion toxin VEGF121/rGel," Journal of Nuclear Medicine, vol. 48, no. 3, pp. 445-454, 2007.

[44] S. R. Cherry, Y. Shao, R. W. Silverman et al., "MicroPET: a high resolution PET scanner for imaging small animals," IEEE Transactions on Nuclear Science, vol. 44, no. 3, pp. 1161-1166, 1997.

[45] A. F. Chatziioannou, S. R. Cherry, Y. Shao et al., "Performance evaluation of microPET: a high-resolution lutetium oxyorthosilicate PET scanner for animal imaging," Journal of Nuclear Medicine, vol. 40, no. 7, pp. 1164-1175, 1999.

[46] D. R. Collingridge, V. A. Carroll, M. Glaser et al., "The development of $\left[{ }^{124} \mathrm{I}\right]$ iodinated-VG76e: a novel tracer for imaging vascular endothelial growth factor in vivo using positron emission tomography," Cancer Research, vol. 62, no. 20, pp. 5912-5919, 2002.

[47] W. Cai, K. Chen, K. A. Mohamedali et al., "PET of vascular endothelial growth factor receptor expression," Journal of Nuclear Medicine, vol. 47, no. 12, pp. 2048-2056, 2006.

[48] A. R. Hsu, W. Cai, A. Veeravagu et al., "Multimodality molecular imaging of glioblastoma growth inhibition with vasculature-targeting fusion toxin $\mathrm{VEGF}_{121} / \mathrm{rGel}$," Journal of Nuclear Medicine, vol. 48, no. 3, pp. 445-454, 2007.

[49] E. Lu, W. R. Wagner, U. Schellenberger et al., "Targeted in vivo labeling of receptors for vascular endothelial growth factor: approach to identification of ischemic tissue," Circulation, vol. 108, no. 1, pp. 97-103, 2003.

[50] M. V. Backer, Z. Levashova, V. Patel et al., "Molecular imaging of VEGF receptors in angiogenic vasculature with single-chain VEGF-based probes," Nature Medicine, vol. 13, no. 4, pp. 504 509, 2007. 
[51] F. G. Blankenberg, S. Mandl, Y. A. Cao et al., "Tumor imaging using a standardized radiolabeled adapter protein docked to vascular endothelial growth factor," Journal of Nuclear Medicine, vol. 45, no. 8, pp. 1373-1380, 2004.

[52] Z. Liu, F. Wang, and X. Chen, "Integrin $\alpha \mathrm{v} \beta 3$-targeted cancer therapy," Drug Development Research, vol. 69, no. 6, pp. 329339, 2008.

[53] B. Jia, J. Shi, Z. Yang et al., " initial evaluation for SPECT imaging of glioma integrin $\alpha \mathrm{v} \beta 3$ expression," Bioconjugate Chemistry, vol. 17, no. 4, pp. 10691076, 2006.

[54] Q. He, Z. Liu, B. Jia et al., "In vivo gamma imaging of the secondary tumors of transplanted human fetal striatum neural stem cells-derived primary tumor cells," NeuroReport, vol. 19, no. 10, pp. 1009-1014, 2008.

[55] Z. Liu, S. Liu, F. Wang, S. Lieu, and X. Chen, "Noninvasive imaging of tumor integrin expression using ${ }^{18} \mathrm{~F}$ - labeled RGD dimer peptide with PEG4 linkers," European Journal of Nuclear Medicine and Molecular Imaging, vol. 36, no. 8, pp. 1296-1307, 2009.

[56] S. Liu, Z. Liu, K. Chen et al., "18 F-labeled galacto and PEGylated RGD dimers for PET imaging of $\alpha \mathrm{v} \beta 3$ integrin expression," Molecular Imaging and Biology, vol. 12, no. 5, pp. 530$538,2010$.

[57] Z. F. Liu, G. Niu, J. Shi et al., "68 Ga-labeled cyclic RGD dimmers with $\mathrm{Gly}_{3} \mathrm{PEG}_{4}$ linkers: promising agents for tumor integrin $\alpha_{\mathrm{v}} \beta_{3}$ PET imaging," European Journal of Nuclear Medicine and Molecular Imaging, vol. 36, pp. 947-957, 2010.

[58] F. Cao, Z. Li, A. Lee et al., "Noninvasive de novo imaging of human embryonic stem cell-derived teratoma formation," Cancer Research, vol. 69, no. 7, pp. 2709-2713, 2009.

[59] A. Veeravagu, Z. Liu, G. Niu et al., "Integrin $\alpha_{v} \beta_{3}$-targeted radioimmunotherapy of glioblastoma multiforme," Clinical Cancer Research, vol. 14, no. 22, pp. 7330-7339, 2008.

[60] Z. F. Liu, B. Jia, H. Y. Zhao, X. Y. Chen, and F. Wang, "Specific targeting of human integrin $\alpha \mathrm{v} \beta 3$ with ${ }^{111}$ In -labeled abegrin $^{\mathrm{TM}}$ in nude mouse models," Molecular Imaging and Biology, vol. 13, no. 1, pp. 112-120, 2011.

[61] Z. Liu, S. Liu, G. Niu, F. Wang, and X. Chen, "Optical imaging of integrin $\alpha_{\mathrm{v}} \beta_{3}$ expression with near- infrared fluorescent RGD dimer with tetra(ethylene glycol) linkers," Molecular Imaging, vol. 9, no. 1, pp. 21-29, 2010.

[62] J. D. Hood and D. A. Cheresh, "Role of integrins in cell invasion and migration," Nature Reviews Cancer, vol. 2, no. 2, pp. 91-100, 2002.

[63] J. P. Xiong, T. Stehle, R. Zhang et al., "Crystal structure of the extracellular segment of integrin $\alpha_{\mathrm{v}} \beta_{3}$ in complex with an ArgGly-Asp ligand," Science, vol. 296, no. 5565, pp. 151-155, 2002.

[64] W. Cai and X. Chen, "Anti-angiogenic cancer therapy based on integrin $\alpha_{\mathrm{v}} \beta_{3}$ antagonism," Anti-Cancer Agents in Medicinal Chemistry, vol. 6, no. 5, pp. 407-428, 2006.

[65] J. P. Xiong, T. Stehle, B. Diefenbach et al., "Crystal structure of the extracettutar segment of integrin $\alpha_{\mathrm{v}} \beta_{3}$," Science, vol. 294, no. 5541, pp. 339-345, 2001.

[66] W. Cai, S. S. Gambhir, and X. Chen, "Multimodality tumor imaging targeting integrin $\alpha_{\mathrm{v}} \beta_{3}$," BioTechniques, vol. 39, no. 6 , pp. S14-S25, 2005.

[67] S. Achilefu, S. Bloch, M. A. Markiewicz et al., "Synergistic effects of light-emitting probes and peptides for targeting and monitoring integrin expression," Proceedings of the National Academy of Sciences of the United States of America, vol. 102, no. 22, pp. 7976-7981, 2005.
[68] Y. Ye, S. Bloch, B. Xu, and S. Achilefu, "Design, synthesis, and evaluation of near infrared fluorescent multimeric RGD peptides for targeting tumors," Journal of Medicinal Chemistry, vol. 49, no. 7, pp. 2268-2275, 2006.

[69] X. B. Ma, J. Tian, C. H. Qin et al., "Early detection of liver cancer based on bioluminescent tomography," Applied Optics, vol. 50, no. 10, pp. 1389-1395, 2011. 

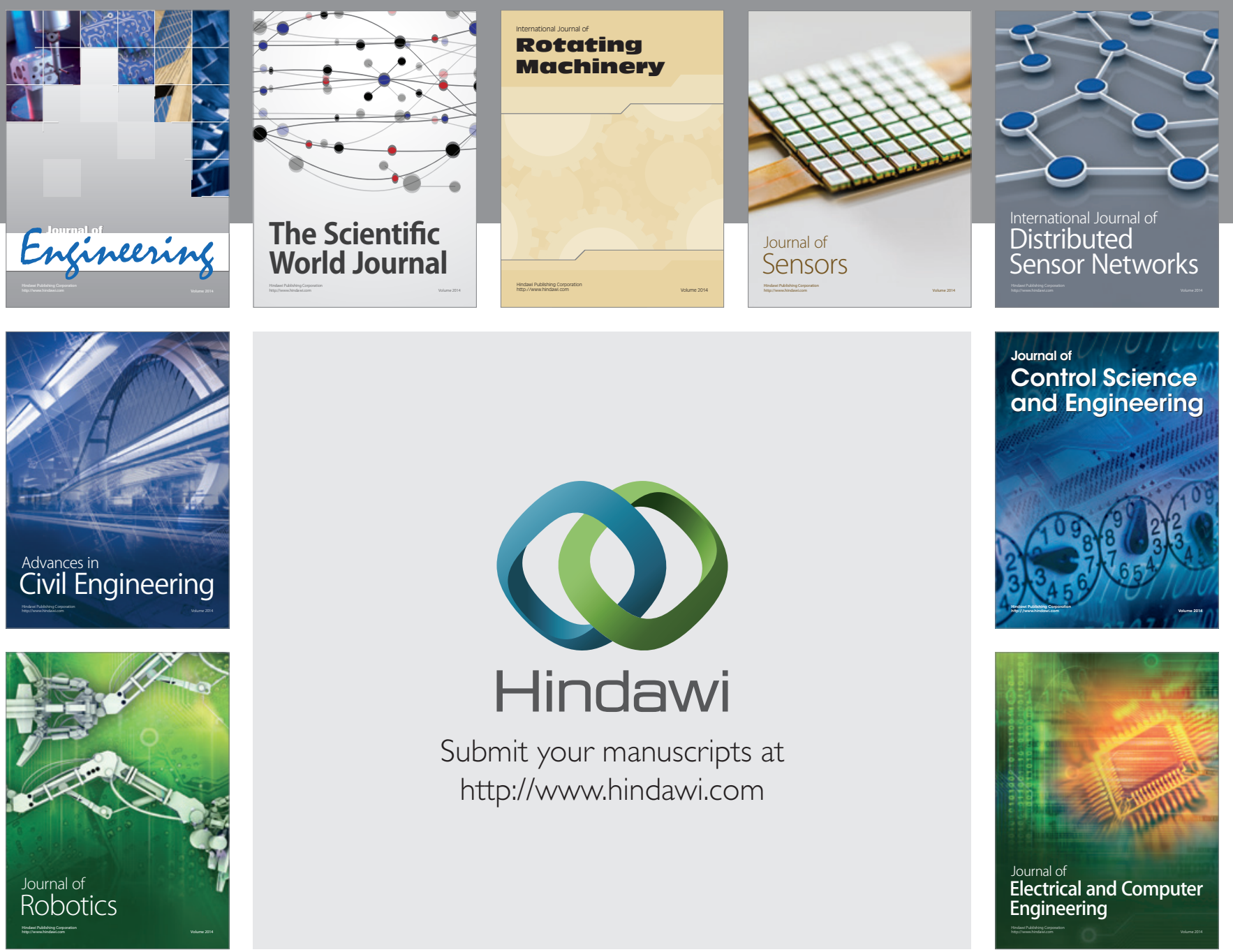

Submit your manuscripts at

http://www.hindawi.com
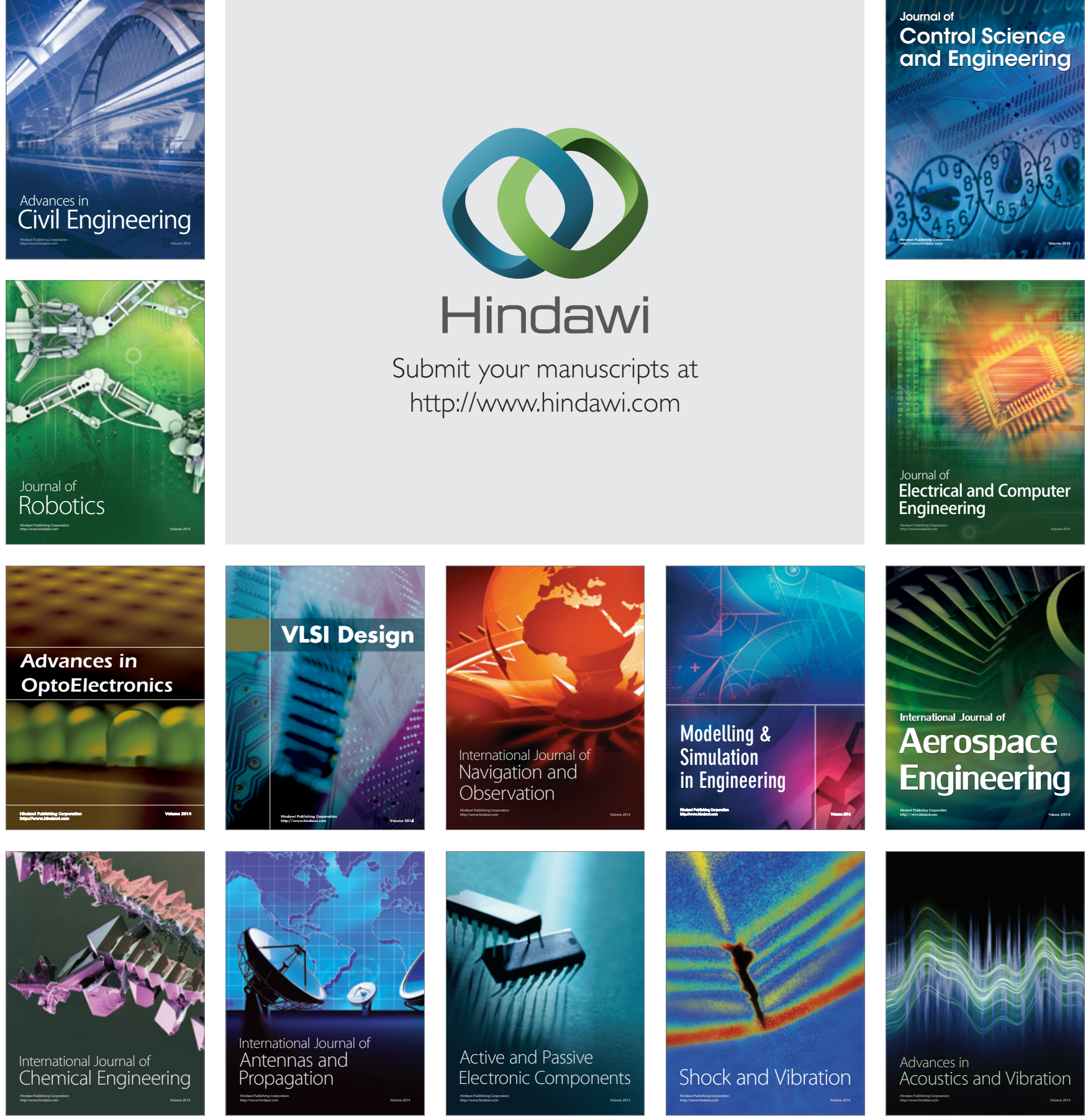